\title{
On weak drop property and quasi-weak drop property
}

\author{
by \\ J. H. QIU (Suzhou)
}

\begin{abstract}
Every weakly sequentially compact convex set in a locally convex space has the weak drop property and every weakly compact convex set has the quasi-weak drop property. An example shows that the quasi-weak drop property is strictly weaker than the weak drop property for closed bounded convex sets in locally convex spaces (even when the spaces are quasi-complete). For closed bounded convex subsets of quasi-complete locally convex spaces, the quasi-weak drop property is equivalent to weak compactness. However, for closed bounded convex sets in sequentially complete locally convex spaces, even the weak drop property does not imply weak compactness. A quasi-complete locally convex space is semi-reflexive if and only if its closed bounded convex subsets have the quasi-weak drop property. For strong duals of quasi-barrelled spaces, semi-reflexivity is equivalent to every closed bounded convex set having the quasi-weak drop property. From this, reflexivity of a quasi-complete, quasi-barrelled space (in particular, a Fréchet space) is characterized by the quasi-weak drop property of the space and of the strong dual.
\end{abstract}

1. Introduction. Let $(X,\|\|)$ be a Banach space and $B(X)$ the closed unit ball $\{x \in X:\|x\| \leq 1\}$. Given $x_{0} \notin B(X)$, the convex hull of $x_{0}$ and $B(X)$ is called the drop generated by $x_{0}$ and denoted by $D\left(x_{0}, B(X)\right)$. Daneš [3] proved that in any Banach space $(X,\|\|)$, for every closed set $A$ at positive distance from $B(X)$, there exists an $x_{0} \in A$ such that $D\left(x_{0}, B(X)\right)$ $\cap A=\left\{x_{0}\right\}$. Modifying the assumption of the Daneš drop theorem, Rolewicz [23] began the study of the drop property for the closed unit ball. He defined the norm \|\| to have the drop property if for every closed set $A$ disjoint from $B(X)$ there exists an $x_{0} \in A$ such that $D\left(x_{0}, B(X)\right) \cap A$ $=\left\{x_{0}\right\}$, and he proved that if the norm \|\| has the drop property then $(X,\|\|)$ is reflexive (see [23, Theorem 5]). Giles, Sims and Yorke [7] defined the following weaker variant: the norm \|\| has the weak drop property if for every weakly sequentially closed set $A$ disjoint from $B(X)$, there exists an $x_{0} \in A$ such that $D\left(x_{0}, B(X)\right) \cap A=\left\{x_{0}\right\}$, and they showed that this property is equivalent to $(X,\|\|)$ being reflexive. Moreover Kutzarova [11] and

2000 Mathematics Subject Classification: 46A55, 46B20.

Key words and phrases: quasi-complete space; reflexive space; quasi-weak drop property; weak drop property; weakly compact set. 
Giles and Kutzarova [6] extended the discussion of the drop and weak drop properties to closed bounded convex sets in Banach spaces. A series of profound results concerning these properties have been obtained (for example, see $[1,2,5-7,11-19,21-24,26]$ and references therein). A closed bounded convex subset $B$ of $X$ is said (see [6]) to have the weak drop property if for every weakly sequentially closed set $A$ disjoint from $B$ there exists an $x_{0} \in A$ such that $D\left(x_{0}, B\right) \cap A=\left\{x_{0}\right\}$. For closed bounded convex sets in Banach spaces, the weak drop property is equivalent to weak compactness (see [6, Theorem 3] or [18, Proposition 4.4.7]).

If we replace "weakly sequentially closed set $A$ " with "weakly closed set $A$ " in the above definition of the weak drop property, then we obtain [22] a new drop property - the quasi-weak drop property, which seems more natural and weaker than the weak drop property. Although a weakly sequentially closed set need not be weakly closed even in a separable Banach space [22, Example 2.2], using streaming sequences introduced by Rolewicz [23], we proved [22, Theorem 2.2] that for closed bounded convex sets in Fréchet spaces (i.e. complete metrizable locally convex spaces), the quasi-weak drop property is equivalent to the weak drop property and both are equivalent to weak compactness (equivalently, weak sequential compactness or weak countable compactness, see [10, p. 318]). From this we know that a Fréchet space is reflexive if and only if every closed bounded convex set in the space has the quasi-weak drop property [22, Corollary 2.1].

In this paper, we prove that in locally convex spaces every weakly sequentially compact convex set has the weak drop property and every weakly compact convex set has the quasi-weak drop property. We construct an example showing that the quasi-weak drop property is strictly weaker than the weak drop property for closed bounded convex sets in locally convex spaces (even when the locally convex spaces are quasi-complete). Moreover we show that for closed bounded convex subsets of quasi-complete locally convex spaces, the quasi-weak drop property is equivalent to weak compactness. Thus a quasi-complete locally convex space is semi-reflexive if and only if its closed bounded convex subsets all have the quasi-weak drop property. However, for closed bounded convex sets in sequentially complete locally convex spaces, even the weak drop property does not imply weak compactness. Since the strong duals of quasi-barrelled spaces are quasi-complete, applying the above criterion for semi-reflexivity we conclude that for strong duals of quasi-barrelled spaces, semi-reflexivity is equivalent to every closed bounded convex set having the quasi-weak drop property. Since a quasicomplete quasi-barrelled space is reflexive if and only if its strong dual is reflexive, we may characterize reflexivity of a quasi-complete quasi-barrelled space (in particular, a Fréchet space) by using the quasi-weak drop property of the space and of the strong dual. 
2. Weak drop property and weak sequential compactness. The concept of the weak drop property for closed bounded convex sets in Banach spaces $([6])$ can be extended to bounded convex sets in locally convex spaces. A bounded convex set $B$ in a locally convex space is said to have the weak drop property if for every weakly sequentially closed set $A$ disjoint from $B$ there exists an $x_{0} \in A$ such that $D\left(x_{0}, B\right) \cap A=\left\{x_{0}\right\}$. We shall see that every weakly sequentially compact convex set in a locally convex space has the weak drop property. In this paper, for a locally convex space $(X, \mathcal{T})$ we denote its topological dual by $X^{*}$ and denote the weak topology on $X$ by $\sigma\left(X, X^{*}\right)$. First we give the following.

Lemma 2.1. Let $(X, \mathcal{T})$ be a locally convex space and $B$ a weakly sequentially compact convex subset of $(X, \mathcal{T})$. Suppose that $A \subset X$ is a weakly sequentially closed set which is disjoint from $B$. Then for any $a_{0} \in A$ and $\varepsilon>0$, there exists $a_{1} \in D\left(a_{0}, B\right) \cap A$ such that

$$
D\left(a_{1}, B\right) \cap A \subset\left\{t a_{1}+(1-t) b: b \in B, 1-\varepsilon<t \leq 1\right\} .
$$

Proof. Denote the absolutely convex hull of the set $\left\{a_{0}\right\} \cup B$ by $W$. Following $[10, \S 20,6(5)]$ we can easily prove that $W$ remains weakly sequentially compact. Clearly, $W$ is a sequentially complete disk. Hence $W$ is a Banach disk (see [20, Corollary 3.2.5]), that is, $\left(\operatorname{sp}[W], p_{W}\right)$ is a Banach space, where $\operatorname{sp}[W]$ denotes the linear span of $W$ and $p_{W}$ denotes the Minkowski gauge of $W$. We observe easily that $D\left(a_{0}, B\right) \cap A-B$ is weakly sequentially compact and that

$$
D\left(a_{0}, B\right) \cap A-B \subset D\left(a_{0}, B\right)-B \subset W-W \subset \operatorname{sp}[W] .
$$

Clearly, the topology on $\operatorname{sp}[W]$ generated by $p_{W}$ is finer than the one induced by the weak topology, hence the weakly sequentially closed set $D\left(a_{0}, B\right) \cap A-B$ is closed in $\left(\operatorname{sp}[W], p_{W}\right)$. Since $A$ is disjoint from $B$, $D\left(a_{0}, B\right) \cap A$ is disjoint from $B$ and $0 \notin D\left(a_{0}, B\right) \cap A-B$. Thus we may assume that

$$
\alpha:=\inf \left\{p_{W}(x-y): x \in D\left(a_{0}, B\right) \cap A, y \in B\right\}>0 .
$$

For $\varepsilon>0$, there exist $a_{1} \in D\left(a_{0}, B\right) \cap A$ and $b_{1} \in B$ such that

$$
p_{W}\left(a_{1}-b_{1}\right)<\alpha(1+\varepsilon) .
$$

Any $x \in D\left(a_{1}, B\right) \cap A$ can be written as

$$
x=t a_{1}+(1-t) b, \quad 0<t \leq 1, b \in B .
$$

Here $t$ cannot be 0 , since otherwise $x=b \in A \cap B$, which contradicts the assumption that $A$ is disjoint from $B$. Since $B$ is convex, $t b_{1}+(1-t) b \in B$ and we have

$$
\alpha \leq p_{W}\left(x-t b_{1}-(1-t) b\right)=p_{W}\left(t a_{1}-t b_{1}\right)=t p_{W}\left(a_{1}-b_{1}\right)<t \alpha(1+\varepsilon) .
$$


From this,

This means that

$$
1<t(1+\varepsilon) \text { and } 1-\varepsilon<\frac{1}{1+\varepsilon}<t \leq 1 .
$$

$$
D\left(a_{1}, B\right) \cap A \subset\left\{t a_{1}+(1-t) b: 1-\varepsilon<t \leq 1, b \in B\right\} .
$$

TheOREm 2.1. Let $(X, \mathcal{T})$ be a locally convex space and $B$ a weakly sequentially compact convex subset of $(X, \mathcal{T})$. Then $B$ has the weak drop property, that is, for any weakly sequentially closed set $A$ disjoint from $B$, there exists $x_{0} \in A$ such that $D\left(x_{0}, B\right) \cap A=\left\{x_{0}\right\}$.

Proof. Take $\varepsilon_{1}>\varepsilon_{2}>\ldots>0$ such that $\sum_{i=1}^{\infty} \varepsilon_{i} \leq 1$. By Lemma 2.1, for any given $a_{0} \in A$ and $\varepsilon_{1}>0$, there exists $a_{1} \in D\left(a_{0}, B\right) \cap A$ such that

$$
D\left(a_{1}, B\right) \cap A \subset\left\{t a_{1}+(1-t) b: 1-\varepsilon_{1}<t \leq 1, b \in B\right\} .
$$

For the above $a_{1} \in A$ and $\varepsilon_{2}>0$, again using Lemma 2.1, there exists $a_{2} \in D\left(a_{1}, B\right) \cap A$ such that

$$
D\left(a_{2}, B\right) \cap A \subset\left\{t a_{2}+(1-t) b: 1-\varepsilon_{2}<t \leq 1, b \in B\right\} .
$$

Repeating this process, we obtain a sequence $\left\{a_{n}\right\} \subset A$ which has the following representations:

$$
\begin{aligned}
a_{1} & =t_{0} a_{0}+\left(1-t_{0}\right) b_{0}, & & \text { where } b_{0} \in B, 0 \leq t_{0} \leq 1 ; \\
a_{n+1} & =t_{n} a_{n}+\left(1-t_{n}\right) b_{n}, & & \text { where } b_{n} \in B, 1-\varepsilon_{n}<t_{n} \leq 1 .
\end{aligned}
$$

If there exists a natural number sequence $n_{1}<n_{2}<\ldots$ such that every $a_{n_{i}}=a_{0}$, then for any $z \in D\left(a_{0}, B\right) \cap A=D\left(a_{n_{i}}, B\right) \cap A$, we have

$$
z=\lambda_{i} a_{n_{i}}+\left(1-\lambda_{i}\right) b_{n_{i}}^{\prime}=\lambda_{i} a_{0}+\left(1-\lambda_{i}\right) b_{n_{i}}^{\prime},
$$

where $b_{n_{i}}^{\prime} \in B$ and $1-\varepsilon_{n_{i}}<\lambda_{i} \leq 1$. Clearly $\lambda_{i} \stackrel{i}{\rightarrow} 1$ and $\left\{b_{n_{i}}^{\prime}\right\}_{i \in \mathbb{N}}$ is a bounded sequence, hence

$$
z=\lim _{i \rightarrow \infty}\left(\lambda_{i} a_{0}+\left(1-\lambda_{i}\right) b_{n_{i}}^{\prime}\right)=a_{0} .
$$

Thus $D\left(a_{0}, B\right) \cap A=\left\{a_{0}\right\}$ and the result is obvious. Now we might as well assume that every $a_{n} \neq a_{0}$. It is easy to see that

$$
\begin{aligned}
& a_{n+1} \\
& \quad=\left(a_{n+1}-a_{n}\right)+\left(a_{n}-a_{n-1}\right)+\ldots+\left(a_{2}-a_{1}\right)+a_{1} \\
& \quad=\left(1-t_{n}\right)\left(b_{n}-a_{n}\right)+\left(1-t_{n-1}\right)\left(b_{n-1}-a_{n-1}\right)+\ldots+\left(1-t_{1}\right)\left(b_{1}-a_{1}\right)+a_{1} .
\end{aligned}
$$

From this,

$$
\begin{aligned}
a_{n+k+1} & -a_{n+1} \\
& =\left(1-t_{n+k}\right)\left(b_{n+k}-a_{n+k}\right)+\ldots+\left(1-t_{n+1}\right)\left(b_{n+1}-a_{n+1}\right) \\
& \in\left(1-t_{n+k}\right)\left(B-D\left(a_{n+k-1}, B\right)\right)+\ldots+\left(1-t_{n+1}\right)\left(B-D\left(a_{n}, B\right)\right) \\
& \subset\left(1-t_{n+k}\right)\left(B-D\left(a_{0}, B\right)\right)+\ldots+\left(1-t_{n+1}\right)\left(B-D\left(a_{0}, B\right)\right) .
\end{aligned}
$$


Remarking that $B-D\left(a_{0}, B\right)$ is a convex set containing 0 , we have

$$
\begin{aligned}
a_{n+k+1}-a_{n+1} & \in\left(\sum_{i=n+1}^{n+k}\left(1-t_{i}\right)\right)\left(B-D\left(a_{0}, B\right)\right) \\
& \subset\left(\sum_{i=n+1}^{n+k} \varepsilon_{i}\right)\left(B-D\left(a_{0}, B\right)\right) \subset 2\left(\sum_{i=n+1}^{n+k} \varepsilon_{i}\right) W,
\end{aligned}
$$

where $W$ denotes the absolutely convex hull of the set $\left\{a_{0}\right\} \cup B$. Since $\sum_{i=1}^{\infty} \varepsilon_{i}<\infty,\left\{a_{n}\right\}_{n \in \mathbb{N}}$ is a Cauchy sequence in $\left(\operatorname{sp}[W], p_{W}\right)$. As seen in the proof of Lemma 2.1, $\left(\operatorname{sp}[W], p_{W}\right)$ is a Banach space, hence there exists an $x_{0} \in \operatorname{sp}[W]$ such that

$$
a_{n} \stackrel{n}{\rightarrow} x_{0} \quad \text { in }\left(\operatorname{sp}[W], p_{W}\right) .
$$

For any fixed $n \in \mathbb{N}$ and every $k \in \mathbb{N}$,

$$
a_{n+k} \in D\left(a_{n+k-1}, B\right) \cap A \subset \ldots \subset D\left(a_{n}, B\right) \cap A .
$$

From $(*), a_{n+k} \stackrel{k}{\rightarrow} x_{0}$ in $\left(X, \sigma\left(X, X^{*}\right)\right)$, and since $D\left(a_{n}, B\right) \cap A$ is weakly sequentially closed, we have

$$
x_{0} \in D\left(a_{n}, B\right) \cap A \subset D\left(a_{0}, B\right) \cap A .
$$

Now for any $z \in D\left(x_{0}, B\right) \cap A$, we conclude that $z \in D\left(a_{n}, B\right) \cap A$. Thus $z$ can be written as follows:

$$
z=\mu_{n} a_{n}+\left(1-\mu_{n}\right) b_{n}, \quad \text { where } b_{n} \in B, 1-\varepsilon_{n}<\mu_{n} \leq 1 .
$$

Hence

$z-a_{n}=\left(1-\mu_{n}\right)\left(b_{n}-a_{n}\right) \in\left(1-\mu_{n}\right)\left(B-D\left(a_{n-1}, B\right)\right) \subset\left(1-\mu_{n}\right)\left(B-D\left(a_{0}, B\right)\right)$.

Since $1-\mu_{n} \stackrel{n}{\rightarrow} 0$ and $B-D\left(a_{0}, B\right)$ is bounded in $(X, \mathcal{T}), z-a_{n} \stackrel{n}{\rightarrow} 0$ in $(X, \mathcal{T})$, or $a_{n} \stackrel{n}{\rightarrow} z$ in $(X, \mathcal{T})$. Combining this with $(*)$, we have $z=x_{0}$, and hence $D\left(x_{0}, B\right) \cap A=\left\{x_{0}\right\}$.

3. Quasi-weak drop property and weak compactness. In [22], we introduced a new drop property as follows.

Definition 3.1 (see [22, Definition 2.1]). Let $B$ be a closed bounded convex set in a locally convex space $X$. If for any weakly closed set $A$ disjoint from $B$, there exists an $x_{0} \in A$ such that $D\left(x_{0}, B\right) \cap A=\left\{x_{0}\right\}$, then $B$ is said to have the quasi-weak drop property.

Next we shall investigate the relationship between the quasi-weak drop property and weak compactness.

TheOrem 3.1. Let $B$ be a weakly compact convex set in a locally convex space $(X, \mathcal{T})$. Then $B$ has the quasi-weak drop property, that is, for 
any weakly closed set $A$ disjoint from $B$, there exists an $x_{0} \in A$ such that $D\left(x_{0}, B\right) \cap A=\left\{x_{0}\right\}$.

Proof. Since $B$ is weakly compact and $A$ is weakly closed, $A-B:=$ $\{x-y: x \in A, y \in B\}$ is weakly closed; see [8, p. 148] or [25, Lemma 6-5-11]. Remarking that $A$ is disjoint from $B$, we have $0 \notin A-B=$ $\operatorname{cl}_{w}(A-B)$, where $\operatorname{cl}_{w}(A-B)$ denotes the weak closure of $A-B$. On the other hand, it is easy to prove that " $B$ is weakly compact" implies " $B$ is locally complete" (on local completeness, see [20, Chapter 5]). By [21, Corollary 3.1], there exists an $x_{0} \in A$ such that $D\left(x_{0}, B\right) \cap A=\left\{x_{0}\right\}$.

Since weak duals of barrelled spaces are quasi-complete (see [8, p. 218] or $[10$, p. 297]), by Theorem 3.1 we have the following.

Corollary 3.1. Let $(X, \mathcal{T})$ be a barrelled space. Then every closed bounded convex set in $\left(X^{*}, \sigma\left(X^{*}, X\right)\right)$ has the quasi-weak drop property.

Proof. Let $B$ be any closed bounded convex set in $\left(X^{*}, \sigma\left(X^{*}, X\right)\right)$. Since $(X, \mathcal{T})$ is barrelled, $B$ is $\sigma\left(X^{*}, X\right)$-compact. By Theorem 3.1, $B$ has the quasi-weak drop property.

For closed bounded convex sets in Fréchet spaces, we proved [22] that the quasi-weak drop property is equivalent to weak compactness, and hence it is equivalent to the weak drop property. But for closed bounded convex sets in locally convex spaces, the quasi-weak drop property and the weak drop property are two different concepts. We construct an example showing that the quasi-weak drop property is strictly weaker than the weak drop property even in quasi-complete locally convex spaces.

Example 3.1. As is well known, $\left(l^{1},\|\|_{1}\right)^{*}=l^{\infty}$. Denote the topological dual of $\left(l^{\infty},\|\|_{\infty}\right)$ by $\left(l^{\infty}\right)^{*}$ and for any $f \in\left(l^{\infty}\right)^{*}$, define $\|f\|:=\sup \{|f(\eta)|$ : $\left.\eta \in l^{\infty},\|\eta\|_{\infty} \leq 1\right\}$. Let $\left(l^{\infty}\right)^{*}$ be endowed with a locally convex topology $\mathcal{T}$ which is finer than the weak topology $\sigma\left(\left(l^{\infty}\right)^{*}, l^{\infty}\right)$ and coarser than the Mackey topology $\tau\left(\left(l^{\infty}\right)^{*}, l^{\infty}\right)$ (in particular, $\mathcal{T}$ may be the weak topology or the Mackey topology). Set $(X, \mathcal{T}):=\left(\left(l^{\infty}\right)^{*}, \mathcal{T}\right)$; then $(X, \mathcal{T})^{*}=l^{\infty}$. Since $\left(l^{\infty},\|\|_{\infty}\right)$ is a barrelled space, $\left(\left(l^{\infty}\right)^{*}, \sigma\left(\left(l^{\infty}\right)^{*}, l^{\infty}\right)\right)$ is quasi-complete and hence $(X, \mathcal{T})=\left(\left(l^{\infty}\right)^{*}, \mathcal{T}\right)$ is also quasi-complete. Denote the closed unit ball in $\left(\left(l^{\infty}\right)^{*},\|\|\right)$ by $B$, that is, $B=\left\{f \in\left(l^{\infty}\right)^{*}:\|f\| \leq 1\right\}$. By the Alaoglu-Bourbaki Theorem, $B$ is $\sigma\left(\left(l^{\infty}\right)^{*}, l^{\infty}\right)$-compact. This means that $B$ is a weakly compact convex set in the quasi-complete locally convex space $(X, \mathcal{T})$. By Theorem 3.1, we conclude that $B$ has the quasi-weak drop property in $(X, \mathcal{T})$. However we shall show that $B$ does not have the weak drop property. By the canonical imbedding from $\left(l^{1},\|\|_{1}\right)$ into $\left(\left(l^{\infty}\right)^{*},\|\|\right)$, we may regard $l^{1}$ as a subspace of $\left(\left(l^{\infty}\right)^{*},\|\|\right)$. We denote by $e_{n}$ the $n$th unit 
vector of $l^{1}$. Put

$$
\begin{aligned}
x_{1} & =2 e_{1}, \\
x_{2} & =e_{1}+\frac{1}{2} e_{2}, \\
x_{3} & =\frac{1}{2} e_{1}+\frac{1}{4} e_{2}+\frac{1}{2} e_{3}, \\
x_{4} & =\frac{1}{4} e_{1}+\frac{1}{8} e_{2}+\frac{1}{4} e_{3}+\frac{1}{2} e_{4},
\end{aligned}
$$

In general,

$$
x_{n}=\frac{1}{2^{n-2}} e_{1}+\frac{1}{2^{n-1}} e_{2}+\frac{1}{2^{n-2}} e_{3}+\ldots+\frac{1}{2} e_{n} \quad \text { for } n \geq 2 .
$$

Clearly,

$$
x_{n+1}=\frac{1}{2} x_{n}+\frac{1}{2} e_{n+1} \in D\left(x_{n}, B\right)
$$

and

$$
\left\|x_{n}\right\|_{1}=\frac{1}{2^{n-2}}+\frac{1}{2^{n-1}}+\frac{1}{2^{n-2}}+\ldots+\frac{1}{2}=1+\frac{1}{2^{n-1}}>1 .
$$

Hence $x_{n} \notin B$ for all $n$. It is easy to observe that

$$
\left\|x_{n}-x_{m}\right\|_{1} \geq \frac{1}{2} \quad \text { for } n \neq m \text {. }
$$

Let $A:=\left\{x_{n}: n \in \mathbb{N}\right\}$. Then $A$ is a closed set in $\left(l^{1},\|\|_{1}\right)$. Since every $\sigma\left(l^{1}, l^{\infty}\right)$-Cauchy sequence in $l^{1}$ is a \|\|$_{1}$-Cauchy sequence (for example, see $\left[10\right.$, p. 281]), the set $A$ is sequentially closed in $\left(\left(l^{\infty}\right)^{*}, \sigma\left(\left(l^{\infty}\right)^{*}, l^{\infty}\right)\right)$. In other words, it is weakly sequentially closed in $(X, \mathcal{T})$. Also, it is disjoint from $B$, as we have previously seen that $\left\|x_{n}\right\|_{1}=\left\|\widehat{x}_{n}\right\|>1$. Since $x_{m} \neq x_{n}$ for $m \neq n$ and $x_{n+1} \in D\left(x_{n}, B\right) \cap A$, we conclude that

$$
D\left(x_{n}, B\right) \cap A \neq\left\{x_{n}\right\} \quad \text { for every } n .
$$

That is, $B$ does not have the weak drop property.

At the end of [22], we proposed the following open problems. Let $B$ be a closed bounded convex set in a locally complete (respectively, sequentially complete, or quasi-complete) locally convex space $(X, \mathcal{T})$. Is the statement " $B$ has the quasi-weak drop property" equivalent to " $B$ is weakly compact"? For a locally complete (respectively, sequentially complete, or quasi-complete) locally convex space $(X, \mathcal{T})$, is the statement "every closed bounded convex subset of $X$ has the quasi-weak drop property" equivalent to " $(X, \mathcal{T})$ is semi-reflexive"? Now we show that the answers to the above problems are affirmative for $(X, \mathcal{T})$ quasi-complete, and negative for $(X, \mathcal{T})$ sequentially complete (or locally complete). Thus the problems are completely solved in these cases. First we recall the following famous result of James. 
Lemma 3.1 (see [4, p. 59] or [9]). In a quasi-complete locally convex space $X$, a weakly closed subset $B$ is weakly compact if and only if every $f \in X^{*}$ attains its supremum on B. Here $X^{*}$ denotes the topological dual of $X$.

Lemma 3.2 (see [22, Lemma 2.1]). Let $\left\{x_{n}\right\}$ be a sequence in a Hausdorff topological space $X$. If $\left\{x_{n}\right\}$ has no cluster point, then the set $A:=\left\{x_{n}\right.$ : $n \in \mathbb{N}\}$ is a closed set.

TheOREM 3.2. Let $(X, \mathcal{T})$ be a quasi-complete locally convex space and $B$ a closed bounded convex subset of $(X, \mathcal{T})$. Then $B$ has the quasi-weak drop property if and only if it is weakly compact.

Proof. By Theorem 3.1, we only need to prove that " $B$ has the quasiweak drop property" implies " $B$ is weakly compact". For any $f \in X^{*} \backslash\{0\}$, let $M:=\sup \{f(x): x \in B\}$. Then $M<\infty$ since $B$ is bounded. Without loss of generality, we may assume that $0 \in B$ and $M>0$. Choose $x_{1} \notin B$ such that

$$
f\left(x_{1}\right)>\frac{4}{3} M
$$

and choose $y_{n} \in B$ such that

$$
f\left(y_{n}\right)>\left(1-\frac{1}{3^{n+1}}\right) M, \quad n \in \mathbb{N} .
$$

We define a sequence $\left\{x_{n}\right\}$ recursively by $x_{n+1}:=\left(x_{n}+y_{n}\right) / 2$. Assume that

$$
f\left(x_{n}\right)>\frac{3^{n}+1}{3^{n}} M .
$$

Then

$$
\begin{aligned}
f\left(x_{n+1}\right) & =\frac{1}{2} f\left(x_{n}\right)+\frac{1}{2} f\left(y_{n}\right)>\frac{1}{2} \cdot \frac{3^{n}+1}{3^{n}} M+\frac{1}{2}\left(1-\frac{1}{3^{n+1}}\right) M \\
& =\frac{1}{2}\left(\frac{3^{n}+1}{3^{n}}+1-\frac{1}{3^{n+1}}\right) M=\frac{3^{n+1}+1}{3^{n+1}} M .
\end{aligned}
$$

By induction we conclude that for every $n$,

$$
f\left(x_{n}\right)>\frac{3^{n}+1}{3^{n}} M>M .
$$

Obviously $x_{n} \notin B$ and $y_{n} \in B$, hence $x_{n} \neq y_{n}$ and $x_{n+1}=\left(x_{n}+y_{n}\right) / 2 \neq x_{n}$. Assume that the sequence $\left\{x_{n}\right\}$ has no weak cluster point. Then the set $A:=\bigcup\left\{x_{n}: n \in \mathbb{N}\right\}$ is weakly closed by Lemma 3.2. By the assumption that $B$ has the quasi-weak drop property, there exists $x_{n} \in A$ such that $D\left(x_{n}, B\right) \cap A=\left\{x_{n}\right\}$. However we also have $x_{n+1} \in D\left(x_{n}, B\right) \cap A$ and $x_{n+1} \neq x_{n}$, a contradiction. Thus $\left\{x_{n}\right\}$ has a weak cluster point $x_{0}$. Hence $\left\{f\left(x_{n}\right)\right\}$ has a cluster point $f\left(x_{0}\right)$. Since $f\left(x_{n}\right)>M$ for all $n$, we conclude that $f\left(x_{0}\right) \geq M$. 
On the other hand,

$$
\begin{aligned}
x_{n+1} & =\frac{x_{n}+y_{n}}{2}=\frac{x_{n-1}}{2^{2}}+\frac{y_{n-1}}{2^{2}}+\frac{y_{n}}{2}=\frac{x_{n-2}}{2^{3}}+\frac{y_{n-2}}{2^{3}}+\frac{y_{n-1}}{2^{2}}+\frac{y_{n}}{2} \\
& =\ldots=\frac{x_{1}}{2^{n}}+\frac{y_{1}}{2^{n}}+\frac{y_{2}}{2^{n-1}}+\ldots+\frac{y_{n}}{2} \\
& =\frac{1}{2^{n}}\left(x_{1}-y_{1}\right)+\left(\frac{y_{1}}{2^{n-1}}+\frac{y_{2}}{2^{n-1}}+\frac{y_{3}}{2^{n-2}}+\ldots+\frac{y_{n}}{2}\right) \\
& =\frac{1}{2^{n}}\left(x_{1}-y_{1}\right)+z_{n+1},
\end{aligned}
$$

where

$$
z_{n+1}=\frac{y_{1}}{2^{n-1}}+\frac{y_{2}}{2^{n-1}}+\frac{y_{3}}{2^{n-2}}+\ldots+\frac{y_{n}}{2} \in \operatorname{conv}\left(\left\{y_{1}, \ldots, y_{n}\right\}\right) \subset B .
$$

Since

$$
x_{n+1}-z_{n+1}=\frac{1}{2^{n}}\left(x_{1}-y_{1}\right) \stackrel{n}{\rightarrow} 0
$$

and $x_{0}$ is a weak cluster point of $\left\{x_{n}\right\}$, it is also a weak cluster point of $\left\{z_{n}\right\}$. Since $z_{n} \in B$ for all $n$ and $B$ is weakly closed, we have $x_{0} \in B$. Hence $f\left(x_{0}\right) \leq M$. Combining this with the previous conclusion that $f\left(x_{0}\right) \geq M$, we have $f\left(x_{0}\right)=M$. By Lemma $3.1, B$ is weakly compact.

From Theorem 3.2, we have the following drop characterization of semireflexive locally convex spaces.

TheOrem 3.3. Let $(X, \mathcal{T})$ be a quasi-complete locally convex space. Then $(X, \mathcal{T})$ is semi-reflexive if and only if every closed bounded convex set in $(X, \mathcal{T})$ has the quasi-weak drop property.

Noting that in Lemma 3.1 the assumption that $X$ is quasi-complete can be replaced by the closed convex hull of $B$ being complete in the Mackey topology $\tau\left(X, X^{*}\right)$ (see $[4$, p. 65$]$ ), we have the following.

Theorem 3.4. Let $(X, \mathcal{T})$ be a locally convex space and $B$ a closed bounded convex subset of $(X, \mathcal{T})$ which is $\tau\left(X, X^{*}\right)$-complete. Then $B$ has the quasi-weak drop property if and only if $B$ is weakly compact.

TheOREm 3.5. Let $(X, \mathcal{T})$ be a locally convex space which is quasi-complete for the Mackey topology $\tau\left(X, X^{*}\right)$. Then $(X, \mathcal{T})$ is semi-reflexive if and only if every closed bounded convex set in $(X, \mathcal{T})$ has the quasi-weak drop property.

However, if we only assume that $(X, \mathcal{T})$ is sequentially complete, the result of Theorem 3.2 is not true anymore. In fact, for closed bounded convex subsets of sequentially complete locally convex spaces, even the weak drop property does not imply weak compactness. 
ExAmple 3.2. Let $\alpha$ run through an index set $\Lambda$ of cardinality $d$. We denote by $l_{d}^{1}$ the vector space of all vectors $x=\left(\xi_{\alpha}\right)$ for which at most countably many coordinates are non-zero and $\sum_{\alpha \in \Lambda}\left|\xi_{\alpha}\right|<\infty$, where all $\xi_{\alpha}$ are real numbers or complex numbers. For any $x=\left(\xi_{\alpha}\right) \in l_{d}^{1}$, define $\|x\|_{1}=\sum_{\alpha \in \Lambda}\left|\xi_{\alpha}\right|$. Then $\left(l_{d}^{1},\|\|_{1}\right)$ is a Banach space with dual $l_{d}^{\infty}$, which consists of all vectors $x=\left(\xi_{\alpha}\right)$ with $\|x\|_{\infty}=\sup _{\alpha \in \Lambda}\left|\xi_{\alpha}\right|<\infty$ (see [10, p. 137]). Suppose that $d>\aleph_{0}$, and denote by $H$ the subspace of $l_{d}^{\infty}$ consisting of all $x=\left(\xi_{\alpha}\right)$ with at most countably many non-zero coordinates $\xi_{\alpha}$. Let $H$ be endowed with any locally convex topology $\mathcal{T}$ which is finer than (or equal to) the weak topology $\sigma\left(H, l_{d}^{1}\right)$ and is coarser than (or equal to) the Mackey topology $\tau\left(H, l_{d}^{1}\right)$. Then $(H, \mathcal{T})$ and $\left(H, \sigma\left(H, l_{d}^{1}\right)\right)$ have the same dual $l_{d}^{1}$ and they have the same closed convex sets. In particular $(H, \mathcal{T})$ has a base of 0-neighborhoods whose members are closed in $\left(H, \sigma\left(H, l_{d}^{1}\right)\right)$. By $[25$, Theorem 6-1-13], $(H, \mathcal{T})$ is sequentially complete if so is $\left(H, \sigma\left(H, l_{d}^{1}\right)\right)$. Let $x^{(n)}=\left(\xi_{\alpha}^{(n)}\right)_{\alpha \in \Lambda}, n=1,2, \ldots$, be a Cauchy sequence in $\left(H, \sigma\left(H, l_{d}^{1}\right)\right)$. Then there exists a countable subset $\left\{\alpha_{1}, \alpha_{2}, \ldots\right\}$ of $\Lambda$ such that

$$
\xi_{\alpha}^{(n)}=0, \quad \forall n \in \mathbb{N}, \forall \alpha \in \Lambda \backslash\left\{\alpha_{1}, \alpha_{2}, \ldots\right\} .
$$

For any fixed $\alpha_{k},\left\{\xi_{\alpha_{k}}^{(n)}\right\}_{n \in \mathbb{N}}$ is a scalar Cauchy sequence, hence there exists $\xi_{\alpha_{k}}^{(0)}$ such that $\xi_{\alpha_{k}}^{(n)} \rightarrow \xi_{\alpha_{k}}^{(0)}$ as $n \rightarrow \infty$. Since $\left\{x^{(n)}\right\}_{n \in \mathbb{N}}$ is a bounded sequence in $\left(l_{d}^{\infty}, \sigma\left(l_{d}^{\infty}, l_{d}^{1}\right)\right)$, and $\left(l_{d}^{\infty},\|\|_{\infty}\right)$ is the strong dual of the Banach space $\left(l_{d}^{1},\|\|_{1}\right)$, by the uniform boundedness principle, there exists $M>0$ such that $\left\|x^{(n)}\right\|_{\infty} \leq M$ for every $n$. That is, $\sup _{n \in \mathbb{N}} \sup _{\alpha \in \Lambda}\left|\xi_{\alpha}^{(n)}\right| \leq M$. Define $x=\left(\xi_{\alpha}\right)$ as follows: $\xi_{\alpha_{k}}=\xi_{\alpha_{k}}^{(0)}$ for $k=1,2, \ldots$, and $\xi_{\alpha}=0$ for any $\alpha \in \Lambda \backslash\left\{\alpha_{1}, \alpha_{2}, \ldots\right\}$. Obviously $x \in H$ and $\|x\|_{\infty} \leq M$. For any fixed $y=\left(\eta_{\alpha}\right)_{\alpha \in \Lambda} \in l_{d}^{1}$, we have

$$
\begin{aligned}
\left|\left\langle x^{(n)}, y\right\rangle-\langle x, y\rangle\right| & =\left|\sum_{\alpha \in \Lambda} \xi_{\alpha}^{(n)} \eta_{\alpha}-\sum_{\alpha \in \Lambda} \xi_{\alpha} \eta_{\alpha}\right| \\
& =\left|\sum_{k=1}^{\infty} \xi_{\alpha_{k}}^{(n)} \eta_{\alpha_{k}}-\sum_{k=1}^{\infty} \xi_{\alpha_{k}} \eta_{\alpha_{k}}\right| \\
& \leq \sum_{k=1}^{\infty}\left|\xi_{\alpha_{k}}^{(n)}-\xi_{\alpha_{k}}^{(0)}\right|\left|\eta_{\alpha_{k}}\right| .
\end{aligned}
$$

For any $\varepsilon>0$, there exists $k_{0} \in \mathbb{N}$ such that $\sum_{k=k_{0}+1}^{\infty}\left|\eta_{\alpha_{k}}\right|<\varepsilon /(4 M+1)$. Since $\xi_{\alpha_{k}}^{(n)} \rightarrow \xi_{\alpha_{k}}^{(0)}$ as $n \rightarrow \infty$, there exists $n_{0} \in \mathbb{N}$ such that

$$
\sum_{k=1}^{k_{0}}\left|\xi_{\alpha_{k}}^{(n)}-\xi_{\alpha_{k}}^{(0)}\right|\left|\eta_{\alpha_{k}}\right|<\frac{\varepsilon}{2}, \quad \forall n \geq n_{0} .
$$


When $n \geq n_{0}$, we have

$$
\begin{aligned}
\left|\left\langle x^{(n)}, y\right\rangle-\langle x, y\rangle\right| & \leq \sum_{k=1}^{\infty}\left|\xi_{\alpha_{k}}^{(n)}-\xi_{\alpha_{k}}^{(0)}\right|\left|\eta_{\alpha_{k}}\right| \\
& =\sum_{k=1}^{k_{0}}\left|\xi_{\alpha_{k}}^{(n)}-\xi_{\alpha_{k}}^{(0)}\right|\left|\eta_{\alpha_{k}}\right|+\sum_{k=k_{0}+1}^{\infty}\left|\xi_{\alpha_{k}}^{(n)}-\xi_{\alpha_{k}}^{(0)}\right|\left|\eta_{\alpha_{k}}\right| \\
& <\frac{\varepsilon}{2}+\frac{\varepsilon}{4 M+1} \cdot 2 M<\varepsilon .
\end{aligned}
$$

That is to say, $x^{(n)} \rightarrow x$ as $n \rightarrow \infty$, in $\left(H, \sigma\left(H, l_{d}^{1}\right)\right)$. Thus $\left(H, \sigma\left(H, l_{d}^{1}\right)\right)$ is sequentially complete. Put

$$
B=\left\{x=\left(\xi_{\alpha}\right) \in H:\|x\|_{\infty}=\sup _{\alpha \in \Lambda}\left|\xi_{\alpha}\right| \leq 1\right\} .
$$

Then clearly $B$ is closed in $\left(H, \sigma\left(H, l_{d}^{1}\right)\right)$ and hence in $(H, \mathcal{T})$. Thus $B$ is a closed bounded convex set in the sequentially complete locally convex space $(H, \mathcal{T})$. We know $[10$, p. 313$]$ that $B$ is $\sigma\left(H, l_{d}^{1}\right)$-sequentially compact but is not $\sigma\left(H, l_{d}^{1}\right)$-compact. By Theorem $2.1, B$ has the weak drop property but it is not weakly compact.

4. Quasi-weak drop property for strong duals. In this section we shall consider the quasi-weak drop property for closed bounded convex sets in strong duals of quasi-barrelled spaces. We shall give a drop characterization of the semi-reflexivity of strong duals of quasi-barrelled spaces. For the notion of quasi-barrelled spaces (i.e. infrabarrelled spaces), we refer to [8, 10, or 25]. First we give the following:

Lemma 4.1. Let $(X, \mathcal{T})$ be a quasi-barrelled space. Then its strong dual $\left(X^{*}, \beta\left(X^{*}, X\right)\right)$ is quasi-complete.

Proof. Let $B$ be any closed bounded set in $\left(X^{*}, \beta\left(X^{*}, X\right)\right)$. Since $(X, \mathcal{T})$ is quasi-barrelled, $B \subset X^{*}$ is $\tau$-equicontinuous and hence relatively $\sigma\left(X^{*}, X\right)$-compact. Assume that $\left\{f_{\delta}\right\} \subset B$ is a $\beta\left(X^{*}, X\right)$-Cauchy net. Then $\left\{f_{\delta}\right\}$ is certainly a $\sigma\left(X^{*}, X\right)$-Cauchy net. Since $B$ is relatively $\sigma\left(X^{*}, X\right)$ compact, there exists $f_{0} \in X^{*}$ such that $f_{\delta} \stackrel{\delta}{\rightarrow} f_{0}$ in $\left(X^{*}, \sigma\left(X^{*}, X\right)\right)$. Because $\left\{f_{\delta}\right\}$ is a $\beta\left(X^{*}, X\right)$-Cauchy net and $\left(X^{*}, \beta\left(X^{*}, X\right)\right)$ has a $\sigma\left(X^{*}, X\right)$ closed 0-neighborhood base, we deduce that $f_{\delta} \stackrel{\delta}{\rightarrow} f_{0}$ in $\left(X^{*}, \beta\left(X^{*}, X\right)\right.$ ) (see [25, Lemma 6-1-11]). Since $B$ is $\beta\left(X^{*}, X\right)$-closed, $f_{0} \in B$. Thus we have shown that $\left(X^{*}, \beta\left(X^{*}, X\right)\right)$ is quasi-complete.

Combining Lemma 4.1 with Theorems 3.2 and 3.3, we obtain immediately the following:

THEOREM 4.1. Let $(X, \mathcal{T})$ be a quasi-barrelled space and $B$ a closed bounded convex subset of $\left(X^{*}, \beta\left(X^{*}, X\right)\right)$. Then $B$ is $\sigma\left(X^{*}, X^{* *}\right)$-compact 
if and only if $B$ has the quasi-weak drop property, i.e. for each $\sigma\left(X^{*}, X^{* *}\right)$ closed set $A$ disjoint from $B$, there exists an $f_{0} \in A$ such that $D\left(f_{0}, B\right) \cap A$ $=\left\{f_{0}\right\}$.

TheOREm 4.2. Let $(X, \mathcal{T})$ be a quasi-barrelled space. Then the strong dual $\left(X^{*}, \beta\left(X^{*}, X\right)\right)$ is semi-reflexive if and only if every closed bounded convex subset of $\left(X^{*}, \beta\left(X^{*}, X\right)\right)$ has the quasi-weak drop property.

If $(X, \mathcal{T})$ is a quasi-complete quasi-barrelled space (in fact, such a space must be barrelled, see $[8$, p. 217] or $[10$, p. 368]), we shall obtain more interesting results. To this end, we will need the following lemmas.

Lemma 4.2 (see [8, p. 229]). The strong dual $\left(X^{*}, \beta\left(X^{*}, X\right)\right)$ of a reflexive space $(X, \mathcal{T})$ is reflexive.

Lemma 4.3 (see [25, Problem 10-2-115]). Let $(X, \mathcal{T})$ be a quasi-complete locally convex space. If $\left(X^{*}, \beta\left(X^{*}, X\right)\right)$ is semi-reflexive, then $(X, \mathcal{T})$ is semi-reflexive.

TheOREM 4.3. Let $(X, \mathcal{T})$ be a quasi-complete quasi-barrelled space (equivalently, a quasi-complete barrelled space). Then the following statements are equivalent:

(i) $(X, \mathcal{T})$ is reflexive

(ii) $\left(X^{*}, \beta\left(X^{*}, X\right)\right)$ is reflexive.

(iii) $\left(X^{*}, \beta\left(X^{*}, X\right)\right)$ is semi-reflexive.

(iv) Every closed bounded convex set in $(X, \mathcal{T})$ has the quasi-weak drop property.

(v) Every closed bounded convex set in $\left(X^{*}, \beta\left(X^{*}, X\right)\right)$ has the quasiweak drop property.

Proof. (i) $\Rightarrow$ (ii). See Lemma 4.2 .

(ii) $\Rightarrow$ (iii). This is obvious.

(iii) $\Rightarrow($ i $)$. By Lemma $4.3,(X, \mathcal{T})$ is semi-reflexive. Also, $(X, \mathcal{T})$ is quasibarrelled. Hence $(X, \mathcal{T})$ is reflexive (see $[8$, p. 229] or $[10$, p. 302]).

(i) $\Leftrightarrow($ iv). Since $(X, \mathcal{T})$ is quasi-complete, by Theorem 3.3 we know that statement (iv) is equivalent to $(X, \mathcal{T})$ being semi-reflexive. And since $(X, \mathcal{T})$ is quasi-barrelled, $(X, \mathcal{T})$ being semi-reflexive is equivalent to it being reflexive.

(iii) $\Leftrightarrow(\mathrm{v})$. This follows from Theorem 4.2.

Obviously a Fréchet space is a quasi-complete, quasi-barrelled space. Moreover we proved [22] that for closed bounded convex subsets of a Fréchet space, the quasi-weak drop property is equivalent to the weak drop property. Therefore we have: 
Corollary 4.1. Let $(X, d)$ be a Fréchet space. Then all the statements in Theorem 4.3 are equivalent, and each is equivalent to the statement that every closed bounded convex set in $(X, d)$ has the weak drop property.

Corollary 4.2. Let $(X,\|\|)$ be a Banach space. Then the following statements are equivalent:

(i) The space $(X,\|\|)$ is reflexive.

(ii) The norm \|\| has the weak drop property.

(iii) The norm |||| has the quasi-weak drop property.

(iv) The strong dual $\left(X^{*},\|\|^{*}\right)$ is reflexive.

(v) The norm \|\|$^{*}$ has the weak drop property.

(vi) The norm \|\|$^{*}$ has the quasi-weak drop property.

Here \|\|$^{*}$ denotes the natural norm on dual $X^{*}$, i.e. $\|f\|^{*}=\sup \{|f(x)|$ : $x \in X,\|x\| \leq 1\}$ for any $f \in X^{*}$.

The author would like to thank the referee for his valuable comments and suggestions.

\section{References}

[1] J. Banaś, On drop property and nearly uniformly smooth Banach spaces, Nonlinear Anal. 14 (1990), 927-933.

[2] L. X. Cheng, Y. C. Zhou and F. Zhang, Danes' drop theorem in locally convex spaces, Proc. Amer. Math. Soc. 124 (1996), 3699-3702.

[3] J. Daneš, A geometric theorem useful in nonlinear functional analysis, Boll. Un. Mat. Ital. 6 (1972), 369-372.

[4] K. Floret, Weakly Compact Sets, Springer, Berlin, 1980.

[5] P. Georgiev, D. Kutzarova and A. Maaden, On the smooth drop property, Nonlinear Anal. 26 (1996), 595-602.

[6] J. R. Giles and D. N. Kutzarova, Characterisation of drop and weak drop properties for closed bounded convex sets, Bull. Austral. Math. Soc. 43 (1991), 377-385.

[7] J. R. Giles, B. Sims and A. C. Yorke, On the drop and weak drop properties for a Banach space, ibid. 41 (1990), 503-507.

[8] J. Horváth, Topological Vector Spaces and Distributions, Vol. 1, Addison-Wesley, Reading, MA, 1966.

[9] R. C. James, Weakly compact sets, Trans. Amer. Math. Soc. 113 (1964), 129-140.

[10] G. Köthe, Topological Vector Spaces I, Springer, New York, 1969.

[11] D. N. Kutzarova, On the drop property of convex sets in Banach spaces, in: Constructive Theory of Functions '87, Sofia, 1988, 283-287.

[12] D. N. Kutzarova and S. Rolewicz, On drop property for convex sets, Arch. Math. (Basel) 56 (1991), 501-511.

[13] P. K. Lin, Some remarks of drop property, Proc. Amer. Math. Soc. 115 (1992), 441-446.

[14] P. K. Lin and X. T. Yu, The weak drop property on closed convex sets, J. Austral. Math. Soc. 56 (A) (1994), 125-130.

[15] V. Montesinos, Drop property equals reflexivity, Studia Math. 87 (1987), 93-100. 
[16] V. Montesinos, A note on drop property of unbounded sets, Arch. Math. (Basel) 57 (1991), 606-608.

[17] W. B. Moors, On a weak ${ }^{*}$ drop property, Bull. Austral. Math. Soc. 47 (1993), 407-414.

[18] D. Pallaschke and S. Rolewicz, Foundations of Mathematical Optimization, Math. Appl. 388, Kluwer, Dordrecht, 1997.

[19] J. P. Penot, The drop theorem, the petal theorem and Ekeland's variational principle, Nonlinear Anal. 10 (1986), 813-822.

[20] P. Pérez Carreras and J. Bonet, Barrelled Locally Convex Spaces, North-Holland, Amsterdam, 1987.

[21] J. H. Qiu, Local completeness and drop theorem, J. Math. Anal. Appl. 266 (2002), 288-297.

[22] - On the quasi-weak drop property, Studia Math. 151 (2002), 187-194.

[23] S. Rolewicz, On drop property, ibid. 85 (1987), 27-35.

[24] —, On A-uniform convexity and drop property, ibid. 87 (1987), 181-191.

[25] A. Wilansky, Modern Methods in Topological Vector Spaces, McGraw-Hill, New York, 1978.

[26] X. Y. Zheng, Drop theorem in topological vector spaces, Chin. Ann. Math. 21 A (2000), 141-148.

Department of Mathematics

Suzhou University

Suzhou, Jiangsu 215006

People's Republic of China

E-mail: qjhsd@sina.com.cn

Received July 31, 2002

Revised version November 12, 2002

(5009) 